\title{
Sparse Coding of Cardiac Signals for Automated Component Selection after Blind Source Separation
}

\author{
D Wedekind ${ }^{1}$, D Kleyko ${ }^{2}$, E Osipov $^{2}$, H Malberg $^{1}$, S Zaunseder ${ }^{1}$, U Wiklund $^{3}$ \\ ${ }^{1}$ Institute of Biomedical Engineering, TU Dresden, Dresden, Germany \\ ${ }^{2}$ Department of Computer Science, Electrical and Space Engineering, Luleå University of \\ Technology, Luleå, Sweden \\ ${ }^{3}$ Department of Biomedical Engineering \& Informatics, Umeå University, Umeå, Sweden
}

\begin{abstract}
Wearable sensor technology like textile electrodes provides novel ambulatory health monitoring solutions but most often goes along with low signal quality. Blind Source Separation (BSS) is capable of extracting the Electrocardiogram (ECG) out of heavily distorted multichannel recordings. However, permutation indeterminacy has to be solved, i.e. the automated selection of the desired BSS output. To that end we propose to exploit the sparsity of the ECG modeled as a spike train of successive heartbeats. A binary code derived from a two-item dictionary $\{$ peak, no peak $\}$ and physiological a-priori information temporally represents every BSS output component. The (best) ECG component is automatically selected based on a modified Hamming distance comparing the components' code with the expected code behavior.

Non-standard ECG recordings from ten healthy subjects performing common motions while wearing a sensor garment were subsequently processed in $10 \mathrm{~s}$ segments with spatio-temporal BSS. Our sparsity-based selection RCODE achieved $98.1 \%$ heart beat detection accuracy (ACC) by selecting a single component each after BSS. Traditional component selection based on higherorder statistics (e.g. skewness) achieved only $67.6 \%$ ACC.
\end{abstract}

\section{Introduction}

Ambulatory vital sign recording supplements the standard clinical data acquisition by long-term measurements for early diagnosis of diseases or health and stress monitoring of people performing potentially dangerous tasks. Using measurement techniques like textile electrodes for wearable sensing, the recorded electrocardiogram (ECG) is of non-standard nature compared to its clinical counterpart. Moreover, the minimal-conductive measurement principle which allows for a flexible health monitoring is also strongly affected by movement artifacts [1].
Blind Source Separation (BSS) is a signal processing technique capable of separating signal mixtures (e.g. mixtures of ECG and distortions) into its constituting components [2]. Spatio-temporal Independent Component Analysis (ICA) based on the FastICA algorithm [2] is one realization of BSS which has shown a superior performance on wearable data compared to the standard ICA [1]. Despite its ability to separate ECG from distortions, ICA is typically only solved up to a permutation (i.e. separated components are available but the output is mostly unordered). Accordingly, a desired output component (e.g. the one best representing the ECG) has to be automatically selected. This selection gains special interest while processing a large number of channels. This is typical for ambulatory multi-channel health recordings and particularly spatio-temporal ICA which adds extra channels during the processing [3].

Two principles for handling permutation indeterminacy have been proposed in the context of ECG processing. The first principle identifies and discards the undesired components (i.e. artifacts) thus indirectly obtaining the ECG component. A combination of second-order and higherorder statistics was used for that purpose in [4] whereas auto-correlative periodicity manifestation was exploited in [5]. Feature decision trees were used to classify artifacts in [6]. The second approach aims at directly identifying the desired component (i.e. the ECG). Again, higher-order statistics have been utilized in [1,7]. Moreover, template matching was applied in [8]. The morphological nature and periodicity of QRS waveforms of the ECG were exploited for instance in $[9,10]$. A combination of both approaches, primarily sorting out undesired BSS components and further selecting the (best) ECG component among the residual channels, each utilizing frequency characteristics, was proposed by our group earlier in [3]. Besides these existing diversified approaches to component selection, an evaluation of their actual selection performance (like in $[3,11])$ is rare. This study proposes a new approach to 


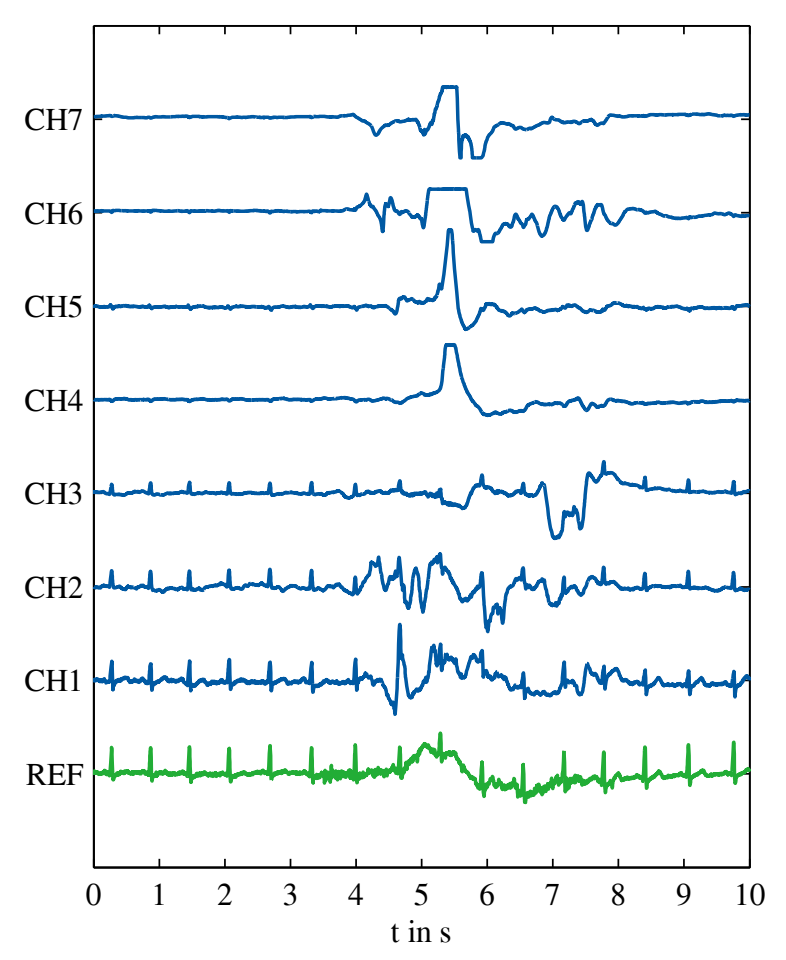

Figure 1. Input data example with heavy distortions and a large motion artifact. REF indicates the conductive reference ECG and $\mathrm{CH} 1-7$ the ECG leads derived from the textile electrodes.

solve the permutation indeterminacy problem including its performance assessment on real data.

Our novel approach aims at directly identifying ECG components in BSS outputs based on a sparse representation of each component. The sparse code itself thereby features QRS waveforms and their temporal behavior, expressed in "spike trains" typical for spatio-temporal BSS on ECG [1]. A comparison is also made with other frequently applied methods which are based on higher-order statistics.

\section{Material and Methods}

\subsection{Wearable Dataset and Processing}

We analyzed recordings from ten healthy subjects wearing a garment with integrated textile electrodes [1] while performing a protocol of motions (standing up, sitting down, walking, flexing chest muscles). Seven ECG leads obtained from the garment (sampling rate $500 \mathrm{~Hz}$ ) were processed in subsequent $10 \mathrm{~s}$ segments (1 s segment shift). A total of (mean \pm standard deviation) $213 \pm$ 8 segments per subject were considered. A reference ECG recorded simultaneously using conductive electrodes



Figure 2. Spatio-temporal BSS output components (excerpt) of the input signals from Fig. 1. Components are vertically ordered according to skewness. Modified Hamming distance $d_{H}$ as well as the sparse code sequence of each output component are shown in grey bars. The RCODE selection is marked orange.

served as ground truth. See Figure 1 for a data example including a large motion artifact. Spatio-temporal BSS using FastICA algorithm with skewness maximization and ten added time lags $(\mathrm{k} \in[0,10])$ [3] was applied to the seven textile ECG leads which resulted in a total of 77 output components per segment. See Figure 2 for an output component excerpt according to the input data shown in Figure 1.

\subsection{Output Component Selection}

Our component selection RCODE consists of three major processing steps: 1) detection of peaks in the output components; 2) coding of the temporal behavior of the peaks of each component; and 3) assessing the components' code for selection of one single component.

The key target for the peak detection is to allow for a sensitive subsequent coding, thus achieving a balance between sensitivity to distortions while maintaining the ability to detect peaks in the presence of distortions. The basic processing steps are indicated in Figure 3. An envelope (ENV) is calculated for each component (BSS) us- 


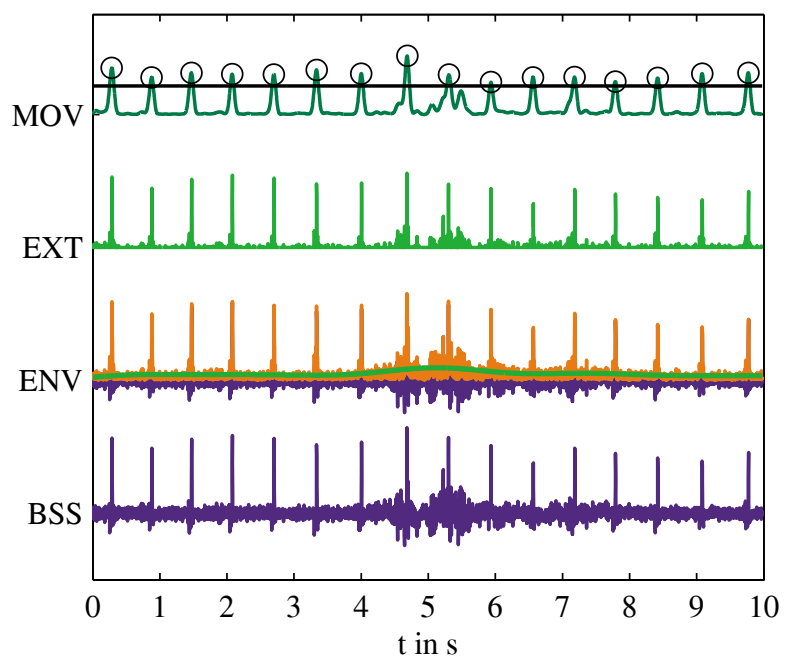

Figure 3. Data processing steps prior to the coding. Spatio-temporal BSS output channel (BSS) including its envelope (orange) and the lowpass-filtered (green) envelope (ENV), the extracted signal (EXT) and its integrated version (MOV) including the threshold (black line) and peak detections (black o).

ing Hilbert transformation. A new signal (EXT) is formed by extracting the signal content above the lowpass-filtered (0.5 Hz 5th-order Butterworth) envelope. This intends to match peak heights between areas with and without distortion. Peaks are further consolidated by moving window integration (MOV) using a 100 ms Hamming window (considered as QRS length [12]). Finally, peaks are detected by applying thresholding using an inversely exponentially weighted average (see black line) on MOV.

The sparse representation of each component's peak detections at times $t_{i}(i \in[1, I], I$ is the number of peak detections) is obtained by coding according to the dictionary $\{$ peak - 1 , no peak - 0$\}$ together with temporal apriori information. The cardiac refractory period $\Delta t_{R}$ is considered as $0.3 \mathrm{~s}$ [10], whereas the maximum peak-topeak distance $\Delta t_{\max }$ is considered as $1.5 \mathrm{~s}$ (i.e. a minimum heart rate of $45 \mathrm{bpm}$ [13]). Accordingly, a sequence $(x) \in\{0,1\}$ is obtained by:

1. $\left(x_{i}\right)=1$ with $i \in[1, I]$

2. add $\left\lceil\left(t_{i+1}-t_{i}\right) / \Delta t_{\max }\right\rceil$ zeros between $x_{i}$ and $x_{i+1}$ if $t_{i+1}-t_{i}>\Delta t_{R}$

3. add $\left\lfloor\left(t_{1}\right) / \Delta t_{\max }\right\rfloor$ zeros to $(\mathrm{x})$ at $t<t_{1}$

4. add $\left\lfloor\left(10-t_{I}\right) / \Delta t_{\max }\right\rfloor$ zeros to $(\mathrm{x})$ at $t>t_{I}$

The sequence $(x)$ of final length $L$ is further assessed by a modified Hamming distance measure $d_{H}$.

$d_{H}$ is designed to indicate the distance from the expected code behavior assuming a perfect ECG component with code $(x)=1,0,1,0, \ldots$ or $(x)=0,1,0,1 \ldots$, respectively. It is constituted by two factors:

$$
d_{H}=w_{d} \cdot d_{10}
$$

where $d_{10}$ is a distance to the expected behavior assessing only pairs of two subsequent code elements $\left(x_{i}, x_{i+1}\right)$ each. It is defined by the ratio between the amount of nondesired code pairs $(0,0)$ or $(1,1)$ and the total amount of code pairs

$$
d_{10}=\frac{\left|\left\{\left(x_{i}, x_{i+1}\right) \mid\left(x_{i}, x_{i+1}\right)=(0,0) \cup(1,1)\right\}\right|}{L-1}
$$

with $i \in[1, L-1]$.

$w_{d}$ weights this distance regarding the length of the longest continuous sequence $\left(x_{i}, x_{i+1}, \ldots\right) \subseteq(x)$ of expected ECG code behavior where all pairs of subsequent code elements $\left(x_{i}, x_{i+1}\right)=(1,0) \cup(0,1)$. Accordingly,

$$
w_{d}=1-\frac{l_{10}}{L-1}
$$

where $l_{10}$ is the length of that longest continuous sequence of the respective $(x)$. If $I=1, d_{H}$ is set to 1 . Examples of code sequences $(x)$ and derived distance measures $d_{H}$ can be seen in Figure 2 .

The component with the minimal $d_{H}$ is selected as RCODE output. In the case of obtaining multiple components with equal minimum $d_{H}$, maximum kurtosis of components' derivative is used to select among them.

In order to compare the traditional component selection based on higher-order statistics [1,4,7], we also used skewness for an automated component selection. Since higherorder statistics are prone to outliers, an outlier-removal using Walsh's non-parametric outlier test [14] was performed on each component prior to selecting the component with the highest skewness (SKEW).

\section{Results}

The performance was evaluated by the heart beat detection accuracy (ACC) [3] obtained by comparing the manual QRS annotations from the reference ECG with the QRS detections using a modified maximum search detector [15]. Figure 4 shows the according boxplots of subject-wise averaged ACC results. The input data quality was described by averaging the ACC for all seven input channels which gave $69.3 \pm 14.2 \%$ (IN). The upper performance limit of a channel selection after BSS was given by manually selecting always the component with the highest ACC which served $99.5 \pm 0.8 \%$ (BEST OUT). The newly proposed automated single channel selection RCODE achieved $98.1 \pm 1.7 \%$ whereas the usage of higher-order statistics in terms of the outlier-adjusted 


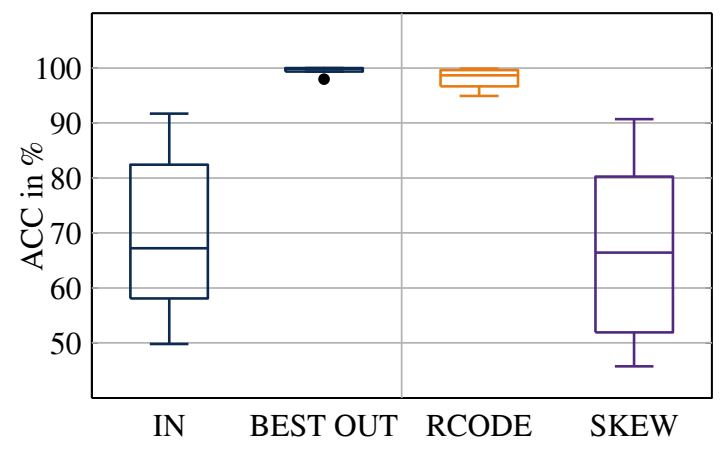

Figure 4. Boxplots showing subject-wise $(\mathrm{N}=10)$ averaged heart beat detection accuracy ACC in \%. Shown are the average input ACC (IN) of seven wearable ECG leads, the manually selected best possible output ACC (BEST OUT) after BSS as well the automated selections RCODE and SKEW. Outliers $(\bullet)$ defined as values exceeding the maximum whisker length ( $1.5 \cdot$ interquartile range).

skewness (SKEW) only reached $67.6 \pm 16.4 \%$. As shown by the skewness ordering in Figure 2, the outlier-adjusted skewness failed to serve as a robust selector (by selecting the component with the maximum skewness SKEW) in the presence of components with distortions. Accordingly, selecting the spatio-temporal BSS output component based on the sparse representation outperformed the traditional method based on higher-order statistics. Moreover, RCODE performed close to the upper selection limit in this particular dataset which will be verified on extended measurements in our future work.

\section{Acknowledgements}

This study was supported by the Swedish Research Council (grant no. 2015-04677). The first author thanks the Graduate Academy of the TU Dresden and the Leonardo-office Saxony (Erasmus+) for funding the short research stay in Umeå, Sweden releasing this contribution.

\section{References}

[1] Wiklund U, Karlsson M, Östlund N, Berglin L, Lindecrantz K, Karlsson S, Sandsjö L. Adaptive spatio-temporal filtering of disturbed ECGs: a multi-channel approach to heartbeat detection in smart clothing. Med Biol Eng Comput 2007;45(6):515-523.

[2] Hyvärinen A. Fast and Robust Fixed-Point Algorithms for Independent Component Analysis. IEEE Trans Neural Netw 1999;10(3):626-634.

[3] Wedekind D, Malberg H, Zaunseder S. Cascaded output selection for processing of capacitive electrocardiograms by means of independent component analysis. In 8th Int.
Workshop on Sensor Data Fusion (SDF). Bonn, Germany, 2013; 1-6.

[4] He T, Clifford G, Tarassenko L. Application of independent component analysis in removing artefacts from the electrocardiogram. Neural Comput Appl 2006;15(2):105-116.

[5] Milanesi M, Martini N, Vanello N, Positano V, Santarelli $\mathrm{M}$, Landini L. Independent component analysis applied to the removal of motion artifacts from electrocardiographic signals. Med Biol Eng Comput 2008;46(3):251-261.

[6] Kuzilek J, Kremen V, Soucek F, Lhotska L. Independent component analysis and decision trees for ECG holter recording de-noising. PLoS ONE 2014;9(6):1-9.

[7] Kuzilek J, Lhotska L. Electrocardiogram beat detection enhancement using Independent Component Analysis. Med Eng Phys 2012;35(6):704-711.

[8] Acharyya A, Maharatna K, Al-Hashimi B, Mondal S. Robust channel identification scheme: solving permutation indeterminacy of ICA for artifacts removal from ECG. In 32nd IEEE Engineering in Medicine and Biology Society (EMBC). Buenos Aires, Argentina, 2010; 1142-1145.

[9] Hamaneh M, Chitravas N, Kaiboriboon K, Lhatoo S, Loparo K. Automated Removal of EKG Artifact From EEG Data Using Independent Component Analysis and Continuous Wavelet Transformation. IEEE Trans Biomed Eng 2014;61(6):1634-1641.

[10] Andreotti F, Riedl M, Himmelsbach T, Wedekind D, Wessel N, Stepan H, Schmieder C, Jank A, Malberg H, Zaunseder S. Robust fetal ECG extraction and detection from abdominal leads. Physiol Meas 2014;35(8):1551-1567.

[11] Wedekind D, Gaetjen F, Rasche S, Matschke K, Malberg H, Zaunseder S. Automated Identification of Cardiac Signals after Blind Source Separation for CameraBased Photoplethysmography. In 35th International Conference on Electronics and Nanotechnology (ELNANO). Kyiv, Ukraine, 2015; 422-427.

[12] Chiarugi F, Sakkalis V, Emmanouilidou D, Kontiris T, Varanini M, Tollis I. Adaptive Threshold QRS Detector with Best Channel Selection Based on a Noise Rating System. In 34th Computers in Cardiology (CinC). Durham, USA, 2007; 157-160.

[13] Christinaki E, Giannakakis G, Chiarugi F, Pediaditis M, Iatraki G. Comparison of Blind Source Separation Algorithms for Optical Heart Rate Monitoring. In 4th Mobihealth. Athens, Greece, 2014; 339-342.

[14] Walsh S. Some Nonparametric Tests of whether the Largest Observations of a Set are too Large or too Small. Annals of Mathematical Statistics 1950;21(4):583-592.

[15] Sameni R. The Open-Source Electrophyiological Toolbox (OSET), version 2.1 (www.oset.ir), 2010.

Address for correspondence:

Daniel Wedekind

TU Dresden

Institute of Biomedical Engineering

01062 Dresden, Germany

daniel.wedekind@tu-dresden.de 